\title{
A Remark on Deformations of Hurwitz Frobenius Manifolds
}

\author{
ALEXANDR BURYAK ${ }^{1,2}$ and SERGEY SHADRIN ${ }^{1,3}$ \\ ${ }^{1}$ Department of Mathematics, University of Amsterdam, P. O. Box 94248, \\ 1090 GE Amsterdam, The Netherlands.e-mail:a.y.buryak@uva.nl; s.shadrin@uva.nl \\ ${ }^{2}$ Department of Mathematics, Moscow State University, Leninskie gory, GSP-2, \\ Moscow 19992, Russia.e-mail: buryaksh@mail.ru \\ ${ }^{3}$ Department of Mathematics, Institute of System Research, Nakhimovsky prospekt 36-1, \\ Moscow117218, Russia.e-mail: shadrin@mccme.ru
}

Received: 4 January 2010 / Revised: 24 June 2010 / Accepted: 29 June 2010

Published online: 10 July 2010 - (C) The Author(s) 2010. This article is published with open access at Springerlink.com

\begin{abstract}
In this note, we use the formalism of multi-KP hierarchies in order to give some general formulas for infinitesimal deformations of solutions of the Darboux-Egoroff system. As an application, we explain how Shramchenko's deformations of Frobenius manifold structures on Hurwitz spaces fit into the general formalism of Givental-van de Leur twisted loop group action on the space of semi-simple Frobenius manifolds.
\end{abstract}

Mathematics Subject Classification (2000). 37K10 (53D45, 37K30).

Keywords. Frobenius manifold, Hurwitz space, Darboux-Egoroff equations, multi-KP hierarchy, deformations.

\section{Introduction}

In [1], Dubrovin has associated a structure of Frobenius manifolds to an arbitrary Hurwitz space of meromorphic functions on Riemann surfaces of genus $g$ with simple finite critical values and a prescribed ramification indices over infinity. Shramchenko [10] observed that the structure of Frobenius manifold associated to a Hurwitz space can be included into a family of Frobenius manifold structures parametrized by a symmetric $g \times g$ matrix. There is a beautiful description of this deformation in terms of the values of holomorphic differentials at the critical points and their $B$-periods matrix.

Meanwhile, Givental [3,4] and, independently, van de Leur [11] have constructed an action of the twisted loop group of $G L_{n}$ on the space of semi-simple Frobenius

A. Buryak was partially supported by the Grants RFBR-07-01-00593, NSh-709.2008.1. Both A. Buryak and S. Shadrin were partly supported by the Vidi grant of NWO. 
manifolds. Moreover, Givental has shown that this group acts transitively on the space semi-simple Frobenius manifolds. This two constructions of the group action were identified in [2] via an identification of the formulas of Y.-P. Lee for the infinitesimal Givental action [7] with the tangent van de Leur action computed in [2] in terms of twisted wave functions of multi-component KP hierarchies.

In this paper, we extend in some way the formulas for the tangent van de Leur action computed in [2]. Namely, we express infinitesimal Lie algebra action on the space of solutions of the Darboux-Egoroff system in terms of the twisted wave functions of multi-component KP. In principle, these formulas are of independent interest. In particular, they allow us to fit Shramchenko's deformations into a general Givental-van de Leur scheme. In particular, it is interesting to trace a correspondence between geometric ingredients of Shramchenko's deformation and particular wave functions of the multi-component KP hierarchy that is associated to an arbitrary solution of the Darboux-Egoroff system in van de Leur's approach.

\subsection{ORGANIZATION OF THE PAPER}

In Section 2, we recall the constructions of Hurwitz Frobenius manifolds and their deformations. In Section 3, we recall the van de Leur approach to Frobenius manifolds and use it in order to derive explicit formulas for the Givental-van de Leur infinitesimal deformations of solutions of the Darboux-Egoroff equations. In Section 4, we discuss the simplest possible example of such infinitesimal deformations that can be integrated explicitly and show that it is exactly the way one could obtain Shramchenko's deformations of Hurwitz Frobenius manifolds.

\section{Frobenius Structures Associated with Hurwitz Spaces}

\subsection{DARBOUX-EGOROFF EQUATIONS}

In this paper, we consider only semi-simple Frobenius manifolds. There is a way to encode the structure of a semi-simple Frobenius manifold in canonical coordinates as a solution of a system of PDEs that is called Darboux-Egoroff equations [1].

Let $n \geq 1$. We consider functions $\gamma_{i j}=\gamma_{j i}, i, j=1, \ldots, n, i \neq j$, in variables $u_{1}, \ldots, u_{n}$. The Darboux-Egoroff equations read:

$$
\begin{aligned}
\frac{\partial \gamma_{i j}}{\partial u_{k}} & =\gamma_{i k} \gamma_{k j}, & & i \neq j \neq k \neq i \\
\sum_{k=1}^{n} \frac{\partial \gamma_{i j}}{\partial u_{k}} & =0 & & \neq j
\end{aligned}
$$

Is it convenient to collect $\gamma_{i j}$ into a symmetric matrix with the diagonal terms that can be either equal to 0 or just arbitrary. We introduce a special notation for that. Let $M$ be a symmetric matrix. By n.d. $M$ we denote the same matrix with non-specified diagonal terms. 
Remark 2.1. A Frobenius manifold in the sense of [1] must be equipped with an Euler vector field. We omit this requirement, since it is not well compatible with the deformation technique that we use this paper.

\subsection{HURWITZ SPACES}

We fix some integer numbers $d_{1}, \ldots, d_{m}>0$ and $g \geq 0$. Let $\mathcal{H}$ be the space of the equivalence classes of the tuples of data $\left(C_{g},\left\{a_{i}, b_{i}\right\}_{i=1}^{g}, f: C_{g} \rightarrow \mathbb{C P}^{1}\right)$, where $C_{g}$ is a Riemann surface of genus $g,\left\{a_{i}, b_{i}\right\}_{i=1}^{g}$ is a choice of the canonical basis of cycles on $C_{g}$, and $f: C_{g} \rightarrow \mathbb{C P}^{1}$ is a meromorphic function of degree $d:=\sum_{i=1}^{m} d_{i}$ with exactly $m$ poles of multiplicity $d_{1}, \ldots, d_{m}$ and $n:=2 g+d+m-2$ simple critical points $x_{1}, \ldots, x_{n} \in C_{g}$. In addition, we choose local parameters $z, \ldots, z_{n}$ at the points $x_{1}, \ldots, x_{n} \in C_{g}$ such that $f=z_{i}^{2}$ in a neighbourhood of $x_{i}$. Two tuples of this data are equivalent if there is a biholomorphic map between two source curves that preserves the rest of the data.

The critical values of meromorphic functions $u_{i}:=f\left(x_{i}\right), i=1, \ldots, n$, are local coordinates on the space $\mathcal{H}$.

We recall the Kokotov-Korotkin construction [5] of a solution of the DarbouxEgoroff equations. Let $W(P, Q)$ be the canonical meromorphic bidifferential on a Riemann surface $C_{g}$. That is, $W(P, Q)$ is specified by the following properties: it is symmetric, it has a quadratic pole on the diagonal $P=Q$ with biresidue 1 , and its $a$-periods with respect to both variables vanish. Then the functions

$$
\gamma_{i j}:=\frac{1}{2} W\left(x_{i}, x_{j}\right):=\left.\frac{1}{2} \frac{W(P, Q)}{d z_{i}(P) d z_{j}(Q)}\right|_{P=x_{i}, Q=x_{j}}
$$

in variables $u_{1}, \ldots, u_{n}$ satisfy the Darboux-Egoroff equations.

\subsection{SHRAMCHENKO'S DEFORMATIONS}

Let $\omega_{i}, i=1, \ldots, g$, be the basis of holomorphic differentials on $C_{g}$ normalized by $\int_{a_{i}} \omega_{j}=\delta_{i j}$. Denote by $\omega$ the matrix of the values of $\omega_{i}$ at critical points:

$$
\omega_{i j}:=\omega_{i}\left(x_{j}\right):=\left.\frac{\omega_{i}(P)}{\mathrm{d} z_{j}(P)}\right|_{P=x_{j}}
$$

Denote by $B$ the matrix of $b$-periods of these differentials divided by $\pi \sqrt{-1}, B_{i j}:=$ $\frac{1}{\pi \sqrt{-1}} \int_{b_{i}} \omega_{j}$. Let $M$ be an arbitrary $g \times g$ symmetric matrix such that $B+M$ is non-degenerate. Shramchenko's [10] deformations of Hurwitz Frobenius manifolds are given by the formula

$$
\text { n.d. } \gamma(M):=\text { n.d. }\left(\gamma-\omega^{t}(B+M)^{-1} \omega\right) \text {. }
$$

Here n.d. $\gamma$ is given by Equation (2). Shramchenko proved that $\gamma_{i j}(M)$ are solutions of the Darboux-Egoroff equations in the variables $u_{1}, \ldots, u_{n}$ in the domain 
$\operatorname{det}(B+M) \neq 0$. Observe that n.d. $\gamma(M)$ tends to n.d. $\gamma$ when $(B+M)^{-1}$ tends to zero.

The proof that n.d. $\gamma(M)$ is a solution of the Darboux-Egoroff equations is based on Rauch variational formula and its corollaries:

$$
\begin{aligned}
\frac{\partial W(P, Q)}{\partial u_{j}} & =\frac{1}{2} W\left(P, x_{j}\right) W\left(Q, x_{j}\right), \\
\frac{\partial \omega_{i}(P)}{\partial u_{j}} & =\frac{1}{2} \omega_{i}\left(x_{j}\right) W\left(P, x_{j}\right), \\
\frac{\partial B_{k l}}{\partial u_{j}} & =\omega_{k}\left(x_{j}\right) \omega_{l}\left(x_{j}\right),
\end{aligned}
$$

where evaluation of differentials at particular points is defined in (2) and (3).

\section{Van de Leur's Formalism for Frobenius Manifolds}

In this section, we explain van de Leur's construction of a Frobenius structure associated with a point in the isotropic semi-infinite Grassmannian.

\subsection{BASIC DEFINITIONS}

Let $V=\left\langle e_{1}, \ldots, e_{n}\right\rangle$ be an $n$-dimensional vector space over $\mathbb{C}$. Let $z$ be a formal variable. We denote by $\mathcal{V}$ the vector space $\Lambda^{\infty / 2}\left(V \otimes \mathbb{C}\left[z^{-1}, z\right]\right)$ spanned by the semi-infinite wegde products

$$
\omega=\left(e_{i_{1}} z^{d_{1}}\right) \wedge\left(e_{i_{2}} z^{d_{2}}\right) \wedge\left(e_{i_{3}} z^{d_{3}}\right) \wedge \cdots
$$

such that the tail of $\omega$ coincides with the tail of vacuum vector

$$
|0\rangle:=\left(e_{1} z^{0}\right) \wedge \cdots \wedge\left(e_{n} z^{0}\right) \wedge\left(e_{1} z^{1}\right) \wedge \cdots \wedge\left(e_{n} z^{1}\right) \wedge \cdots .
$$

By tail of $\omega$ we call another basis vector in $\mathcal{V}$ that is obtained from $\omega$ by removing the first few factors in the wedge product.

Consider a matrix series $A(z) \in \operatorname{End}(V) \otimes \mathbb{C}\left[\left[z^{-1}, z\right]\right]$ such that $A^{t}(-z) A(z)=$ Id $\cdot z^{0}$ (it is better to imagine it as a finite product of invertible matrix series in $\operatorname{End}(V) \otimes \mathbb{C}[[z]]$ and $\operatorname{End}(V) \otimes \mathbb{C}\left[\left[z^{-1}\right]\right]$ with the unit constant term satisfying the same symplectic condition).

Let $\alpha_{i}$ be a local Lie algebra element whose action on $V \otimes \mathbb{C}\left[z^{-1}, z\right]$ is defined by

$$
\alpha_{i}\left(e_{j} z^{d}\right):= \begin{cases}e_{j} z^{d+1} & \text { if } i=j, \\ 0 & \text { otherwise }\end{cases}
$$

and is expanded to $\mathcal{V}$ by the Leibnitz rule. 
All basic objects that we are going to consider are some matrix elements of the operator

$$
\mathcal{A}:=\exp \left(\sum_{i=1}^{n} \alpha_{i} u_{i}\right) A(z),
$$

where $u_{1}, \ldots, u_{n}$ are formal variables.

We denote by $\gamma_{i j}=\gamma_{i j}(A), i, j=1, \ldots, n, i \neq j$, the following matrix elements of $\mathcal{A}$ :

$$
\gamma_{i j}:= \pm \frac{\left.\langle\mid 0\rangle|\mathcal{A}|\left(e_{i} z^{-1}\right) \wedge \partial_{\left(e_{j} z^{0}\right)}|0\rangle\right)}{\langle\mid 0\rangle|\mathcal{A} \| 0\rangle\rangle}
$$

(the vector $\left(e_{i} z^{-1}\right) \wedge \partial_{\left(e_{j} z^{0}\right)}|0\rangle$ is obtained, up to a sign, from the vacuum vector $|0\rangle$ by the replacement of $\left(e_{j} z^{0}\right)$ by $\left.\left(e_{i} z^{-1}\right)\right)$.

We denote by $\left(\Psi_{d}\right)_{i j}=\left(\Psi_{d}\right)_{i j}(A), i, j=1, \ldots, n$, the following matrix elements of $\mathcal{A}$ :

$$
\left(\Psi_{d}\right)_{i j}:=\frac{\left.\left\langle\left(e_{j} z^{-1}\right) \wedge \mid 0\right\rangle|\mathcal{A}|\left(e_{i} z^{-1-d}\right) \wedge|0\rangle\right\rangle}{\langle\mid 0\rangle|\mathcal{A} \| 0\rangle\rangle} .
$$

These matrices can be arranged into a generating series $\Psi(z):=\sum_{d=0}^{\infty} z^{d} \Psi_{d}$ that would be a wave function of multi-KP hierarchy multiplied by $A(z)$ from the right. The property $A^{t}(-z) A(z)=\mathrm{Id} \cdot z^{0}$ imply that $\Psi^{t}(-z) \Psi(z)=\mathrm{Id} \cdot z^{0}$.

Van de Leur has shown in [11] that a formal locally semi-simple Frobenius structure can expressed in terms of the matrices $\gamma$ and $\Psi_{d}, d \geq 0$. In particular, $\gamma_{i j}$ is a solution of the Darboux-Egoroff system; $u_{1}, \ldots, u_{n}$ are canonical coordinates; $\Psi_{0}^{t} \Psi_{1} \mathbf{1}$ is a column of flat coordinates (here 1 is the column of units); and (1/2) . $\mathbf{1}^{t} \Psi_{0}^{t}\left(-\Psi_{3} \Psi_{0}^{t}+\Psi_{2} \Psi_{1}^{t}\right) \Psi_{0} \mathbf{1}$ is the prepotential of a Frobenius manifold.

\subsection{INFINITESIMAL DEFORMATIONS}

From the previous section we see that the right multiplication of a matrix $A(z)$ by a matrix $B(z)$ defines the action on the functions $\gamma(A), \Psi_{d}(A)$ of the groups of matrices $B(z) \in \operatorname{Hom}(V, V) \otimes \mathbb{C}[[z]], B^{t}(-z) B(z)=\mathrm{Id} \cdot z^{0}$, and $B\left(z^{-1}\right) \in$ $\operatorname{Hom}(V, V) \otimes \mathbb{C}\left[\left[z^{-1}\right]\right], B^{t}\left(-z^{-1}\right) B\left(z^{-1}\right)=\mathrm{Id} \cdot z^{0}$, both with the unit constant term. This group action is crucially important, see, e.g., [2, Introduction] for a list of particular applications and references.

We discuss the corresponding Lie algebra action. Let $k \geq 0$ and $\ell>0$. Let matrices $r$ and $s$ be symmetric for odd $\ell$ and skewsymmetric for even $\ell$. It is proven in [2] that 


$$
\begin{aligned}
& \left.\frac{\partial}{\partial \epsilon}\right|_{\epsilon=0} \Psi_{k}\left(A \exp \epsilon\left(r z^{-\ell}\right)\right) \\
& \quad=\Psi_{\ell+k}(A) r-\sum_{p=1}^{\ell} \sum_{q=0}^{\ell-p}(-1)^{\ell-p-q} \Psi_{q}(A) r \Psi_{\ell-p-q}^{t}(A) \Psi_{p+k}(A) ; \\
& \left.\frac{\partial}{\partial \epsilon}\right|_{\epsilon=0} \Psi_{k}\left(A \exp \epsilon\left(s z^{\ell}\right)\right)= \begin{cases}\Psi_{k-\ell}(A) s, & \ell \leq k ; \\
0, & \ell>k\end{cases}
\end{aligned}
$$

This allows to compute the action of this Lie algebra on the prepotential of Frobenius manifolds in flat coordinates, since both the prepotential and the flat coordinates are expressed in terms of $\Psi_{d}, d \geq 0$.

In order to deal with Hurwitz Frobenius manifolds, we need some formulas for the Lie algebra action on n.d. $\gamma(A)$.

THEOREM 3.1. Let $\ell \geq 0$. Let matrices $r$ and $s$ be symmetric for odd $\ell$ and skewsymmetric for even $\ell$. We have:

$$
\begin{aligned}
& \left.\frac{\partial}{\partial \epsilon}\right|_{\epsilon=0} \text { n.d. } \gamma\left(A \exp \epsilon\left(r z^{-\ell}\right)\right)=\text { n.d. } \sum_{i+j=\ell-1}(-1)^{j-1} \Psi_{i}(A) r \Psi_{j}^{t}(A) \\
& \left.\frac{\partial}{\partial \epsilon}\right|_{\epsilon=0} \text { n.d. } \gamma\left(A \exp \epsilon\left(s z^{\ell}\right)\right)=0 .
\end{aligned}
$$

Proof. This theorem is an easy consequence of formulas (8) and (9). Indeed, there is a relation between $\Psi_{d}, d \geq 0$ and n.d. $\gamma$ that is proven in [11]. For any $d \geq 0, k=1, \ldots, n$, we have:

$$
\frac{\partial}{\partial u_{k}} \Psi_{d}=E_{k k} \Psi_{d-1}+\left[\text { n.d. } \gamma, E_{k k}\right] \Psi_{d}
$$

Here and below we assume that $\Psi_{-1}=0$ and we use $\gamma$ with arbitrary diagonal terms since they disappear in the commutator with $E_{k k}$. By $E_{k k}$ we denote the matrix unit, that is $\left(E_{k k}\right)_{i j}:=\delta_{i k} \delta_{j k}$. This formula gives an expression for all elements of n.d. $\gamma$ in terms of $\Psi_{0}$ and its derivatives.

From (12) it follows that

$$
\begin{aligned}
\left.\frac{\partial}{\partial \epsilon}\right|_{\epsilon=0} \frac{\partial}{\partial u_{k}} \Psi_{0}\left(A \exp \epsilon\left(r z^{-\ell}\right)\right)= & {\left[\left.\frac{\partial}{\partial \epsilon}\right|_{\epsilon=0} \gamma\left(A \exp \epsilon\left(r z^{-\ell}\right)\right), E_{k k}\right] \Psi_{0}(A) } \\
& +\left.\left[\gamma(A), E_{k k}\right] \frac{\partial}{\partial \epsilon}\right|_{\epsilon=0} \Psi_{0}\left(A \exp \epsilon\left(r z^{-\ell}\right)\right)
\end{aligned}
$$


Hence, using (8), we obtain the following equation:

$$
\begin{aligned}
& \frac{\partial}{\partial u_{k}}\left(\Psi_{\ell} r+\sum_{q=0}^{\ell-1}(-1)^{\ell-q} \Psi_{q} r \Psi_{\ell-q}^{t} \Psi_{0}\right) \\
& \quad=\left[\delta \gamma, E_{k k}\right] \Psi_{0}+\left[\gamma, E_{k k}\right]\left(\Psi_{\ell} r+\sum_{q=0}^{\ell-1}(-1)^{\ell-q} \Psi_{q} r \Psi_{\ell-q}^{t} \Psi_{0}\right),
\end{aligned}
$$

where we denote $\left.\frac{\partial}{\partial \epsilon}\right|_{\epsilon=0} \gamma\left(A \exp \epsilon\left(r z^{-\ell}\right)\right)$ by $\delta \gamma$. Using Equation (12) and $\Psi^{t}(-z) \Psi(z)=\mathrm{Id} \cdot z^{0}$, we see that

$$
\left[\delta \gamma, E_{k k}\right] \Psi_{0}=\left[E_{k k}, \sum_{i=0}^{\ell-1}(-1)^{i} \Psi_{\ell-1-i} r \Psi_{i}^{t}\right] \Psi_{0} .
$$

Since $\Psi_{0}$ is invertible, $\Psi_{0} \Psi_{0}^{t}=\mathrm{Id}$, we obtain Equation (10). Equation (11) can be proven in the same way, but in fact it is obvious from the definition of $\gamma$.

EXAMPLE 3.2. The simplest non-trivial deformation would be by an element $r z^{-1}$, where $r$ is an arbitrary symmetric matrix. Denote by $\delta_{r} \gamma$ and $\delta_{r} \Psi_{d}$ the corresponding infinitesimal deformations. We have the following system of equations:

$$
\begin{aligned}
\text { n.d. } \delta_{r} \gamma & =- \text { n.d. } \Psi_{0} r \Psi_{0}^{t} \\
\delta_{r} \Psi_{0} & =\Psi_{1} r-\Psi_{0} r \Psi_{0}^{t} \Psi_{1} \\
\delta_{r} \Psi_{1} & =\Psi_{2} r-\Psi_{0} r \Psi_{0}^{t} \Psi_{2} \\
& \text { and so on. }
\end{aligned}
$$

\section{Special Deformations}

Shramchenko's deformations of Hurwitz Frobenius manifolds discussed in Section 2.3 fits into a special case of Example 3.2 that can be integrated explicitly.

\subsection{THE INPUT}

Consider a symmetric $n \times n$ matrix n.d. $\gamma$ whose elements are functions in $u_{1}, \ldots, u_{n}$. Let n.d. $\gamma$ be a solution of the Darboux-Egoroff equations (1). There are two matrix-valued functions with clear geometric meanings associated with the Frobenius structures corresponding to n.d. $\gamma$.

First, there is a solution of the commutativity equations [8], which is a symmetric $n \times n$ matrix $C=C\left(u_{1}, \ldots, u_{n}\right)$ such that $d C \wedge d C=0$. In terms of multi-KP tau-functions, $C=\Psi_{0}^{t} \Psi_{1}$.

Second, one can consider $\Psi_{0}$ itself. In geometric terms $\Psi_{0}$ is defined by the equation $\mathrm{d} C=\Psi_{0}^{t} \cdot \operatorname{diag}\left(\mathrm{d} u_{1}, \ldots, \mathrm{d} u_{n}\right) \cdot \Psi_{0}$. That is, it is the matrix that relates 
the canonical and the flat coordinates for the underlying Frobenius structures, see $[1,11]$. An alternative way to define $\Psi_{0}$ is the following. Consider the system of equations (it is equivalent to Equation (12) with $d=0$ ):

$$
\begin{aligned}
\frac{\partial\left(\Psi_{0}\right)_{i j}}{\partial u_{k}} & =\gamma_{i k}\left(\Psi_{0}\right)_{k j}, \quad i \neq k, \\
\sum_{k=1}^{n} \frac{\partial\left(\Psi_{0}\right)_{i j}}{\partial u_{k}} & =0 .
\end{aligned}
$$

Compatibility of this system of equations follows from the Darboux-Egoroff equations for n.d. $\gamma$. This system of equations implies that $\partial\left(\Psi_{0}^{t} \Psi_{0}\right) / \partial u_{k}=0, k=$ $1, \ldots, n$, and $\Psi_{0}$ that we need is a particular solution of this system of equations such that $\Psi_{0}^{t} \Psi_{0}=$ id.

\subsection{SPECIAL DEFORMATIONS}

We consider a distribution of vector subspaces in the tangent bundle of the space of the solutions of the Darboux-Egoroff equations. It is given by the Giventalvan de Leur tangent vectors of the type (13) described in Example 3.2. It is easy to see that, roughly speaking, a deformation of a particular solution of the DarbouxEgoroff equations is given by an ordinary differential equations of the infinite order.

However, there is a special class of infinitesimal deformations that can be reduced to a finite order ODEs. We fix a positive integer $g \leq n / 2$. Let $D$ be a $g \times n$ constant matrix of rank $g$ such that $D D^{t}=0$. Let us consider the distribution in the tangent bundle of the moduli space of the solutions of the DarbouxEgoroff equations given by the Givental-van de Leur tangent vectors of the type (13) described in Example 3.2 with the matrix $r$ that can be represented as $r=D^{t} M D$, where $M$ is an arbitrary symmetric $g \times g$ matrix. In that case, Equation (13) can be reduced to an ODE of finite order.

PROPOSITION 4.1 Equation (13) for the matrix $r=D^{t} M D$ implies the following system of $O D E s$ for n.d. $\gamma, \omega:=D \Psi_{0}^{t}$, and $B:=D C D^{t}$ :

$$
\begin{aligned}
\text { n.d. } \delta_{M} \gamma & =- \text { n.d. } \omega^{t} M \omega ; \\
\delta_{M} \omega & =-B M \omega ; \\
\delta_{M} B & =-B M B .
\end{aligned}
$$

Proof Direct computation.

In order to use this proposition for a particular n.d. $\gamma$ without going back to the full multi-KP framework, we need an independent definitions of $\omega$ and $B$ in terms of $\gamma$ and $D$. We define $\omega$ as a $g \times n$-matrix-valued solutions of the equation

$$
\mathrm{d} \omega=\omega \cdot\left[\operatorname{diag}\left(\mathrm{d} u_{1}, \ldots, \mathrm{d} u_{n}\right), \text { n.d. } \gamma\right]
$$


with the constant term $\left.\omega\right|_{u=0}=\left.D \Psi_{0}^{t}\right|_{u=0}$. We define $B$ to be a $g \times g$-matrix-valued solution of the equation

$$
\mathrm{d} B=\omega \cdot \operatorname{diag}\left(\mathrm{d} u_{1}, \ldots, \mathrm{d} u_{n}\right) \cdot \omega^{t}
$$

with the constant term $\left.B\right|_{u=0}=\left.D C D^{t}\right|_{u=0}$.

Equations (16) can be integrated explicitly in the case when $M$ is a constant matrix independent of n.d. $\gamma, \omega$, and $B$. Indeed, let us define n.d. $\gamma(\epsilon), \omega(\epsilon)$, and $B(\epsilon)$ by the following formulas:

$$
\begin{aligned}
\text { n.d. } \gamma(\epsilon) & :=\text { n.d. } \gamma-\text { n.d. } \omega^{t} \epsilon M(1+\epsilon B M)^{-1} \omega ; \\
\omega(\epsilon) & =(1+\epsilon B M)^{-1} \omega ; \\
B(\epsilon) & =(1+\epsilon B M)^{-1} B .
\end{aligned}
$$

(these formulas are defined in the domain where $(1+\epsilon B M)$ is invertible).

PROPOSITION 4.2 The matrices n.d. $\gamma(\epsilon), \omega(\epsilon)$, and B( $\epsilon)$ satisfy Equations (17) and (18) for any $\epsilon \geq 0$. They integrate the constant vector field determined by the matrix $M$, that is,

$$
\begin{aligned}
\text { n.d. } \frac{\partial \gamma(\epsilon)}{\partial \epsilon} & =- \text { n.d. } \omega(\epsilon)^{t} M \omega(\epsilon) ; & \text { n.d. }\left.\gamma(\epsilon)\right|_{\epsilon=0} & =\text { n.d. } \gamma ; \\
\frac{\partial \omega(\epsilon)}{\partial \epsilon} & =-B(\epsilon) M \omega(\epsilon) ; & \left.\omega(\epsilon)\right|_{\epsilon=0} & =\omega ; \\
\frac{\partial B(\epsilon)}{\partial \epsilon} & =-B(\epsilon) M B(\epsilon) ; & \left.B(\epsilon)\right|_{\epsilon=0} & =B ;
\end{aligned}
$$

Proof Direct computation.

\subsection{SHRAMCHENKO'S FORMULAS}

In this context, Shramchenko's formulas are a version of formulas (19) for $\epsilon=1$, with some appropriate changes. Let us use n.d. $\gamma, \omega$ and $B$ defined in Section 2.3. Equations (5)-(7) imply Darboux-Egoroff equations for n.d. $\gamma$ and Equations (17)(18). Therefore, we have a system suitable for deformation given by (16) (the initial conditions for $\omega$ and $B$ depend on the choice of a particular point of a formal expansion). Indeed, let us substitute $\epsilon=1$ in Equation (19). We have:

$$
\text { n.d. }\left.\gamma(\epsilon)\right|_{\epsilon=1}=\text { n.d. }\left.\gamma(\epsilon)\right|_{\epsilon=0}-\text { n.d. } \omega^{t}\left(M^{-1}+B\right)^{-1} \omega .
$$

If we change the notations in order to replace $M^{-1}$ with $M$, we obtain exactly formula (4).

Remark 4.3. One could obtain the same solution of the Darboux-Egoroff equations from a special deformation of the following triple: n.d. $\tilde{\gamma}:=\gamma-\omega^{t} B \omega, \tilde{\omega}:=$ $B^{-1} \omega$, and $\tilde{B}:=B^{-1}$. In that case some formulas would look a bit simpler. 
Remark 4.4. Deformations of "real doubles" [9] of Hurwitz Frobenius manifolds fit into exactly the same scheme as we discuss in Section 4.2.

Moreover, in our argument there is still some freedom for the choice of the matrix $D$. For example, one can look for the deformations corresponding to a matrix $D$ represented as $D:=U D_{0}$, where $U$ is an arbitrary $g \times g$ matrix and $D_{0}$ is some fixed $g \times n$ matrix that satisfies $D_{0} D_{0}^{t}=0$. It would give us a family of special deformations parametrized by two matrices, and the deformations of Hurwitz Frobenius manifolds considered in [6, Remark 4] fit into this scheme after a change of parametrization.

\section{Acknowledgements}

The authors are grateful to J. van de Leur and H. Posthuma for useful remarks and discussions and to an anonymous referee for a number of suggestions that helped us to improve the exposition.

Open Access This article is distributed under the terms of the Creative Commons Attribution Noncommercial License which permits any noncommercial use, distribution, and reproduction in any medium, provided the original author(s) and source are credited.

\section{References}

1. Dubrovin, B.: Geometry of 2D topological field theories. In: Integrable Systems and Quantum Groups (Montecatini Terme, 1993). Lecture Notes in Mathematics, vol. 1620, pp. 120-348. Springer, Berlin (1996)

2. Feigin, E., van de Leur, J., Shadrin, S.: Givental symmetries of Frobenius manifolds and multi-component KP tau-functions. Adv. Math. 224(3), 1031-1056 (2010)

3. Givental, A.: Semisimple Frobenius structures at higher genus. Int. Math. Res. Notices 2001(23), 1265-1286 (2001)

4. Givental, A.: Gromov-Witten invariants and quantization of quadratic Hamiltonians. Mosc. Math. J. 1(4), 551-568 (2001)

5. Kokotov, A., Korotkin, D.: A new hierarchy of integrable systems associated to Hurwitz spaces. Philos. Trans. R. Soc. Lond. Ser. A Math. Phys. Eng. Sci. 66(1867), 1055-1088 (2008)

6. Korotkin, D., Shramchenko, V.: Riemann-Hilbert problem for Hurwitz Frobenius manifolds: regular singularities. arXiv:0909.0543v2, 1-61

7. Lee, Y.-P.: Invariance of tautological equations I: conjectures and applications. J. Eur. Math. Soc. (JEMS) 10(2), 399-413 (2008)

8. Shadrin, S., Zvonkine, D.: A group action on Losev-Manin cohomological field theories. arXiv:0909.0800v1, 1-21

9. Shramchenko, V.: "Real doubles" of Hurwitz Frobenius manifolds. Comm. Math. Phys. 256(3), 635-680 (2005)

10. Shramchenko, V.: Deformations of Frobenius structures on Hurwitz spaces. Int. Math. Res. Notices 2005(6), 339-387 (2005)

11. van de Leur, J.: Twisted $\mathrm{GL}_{n}$ loop group orbit and solutions of the WDVV equations. Int. Math. Res. Notices 2001(11), 551-573 